\title{
Decomposing Oriented Graphs into Six Locally Irregular Oriented Graphs
}

\author{
Bensmail, Julien; Renault, Gabriel
}

Published in:

Graphs and Combinatorics

Link to article, DOI:

$10.1007 / \mathrm{s} 00373-016-1705-\mathrm{z}$

Publication date:

2016

Document Version

Peer reviewed version

Link back to DTU Orbit

Citation (APA):

Bensmail, J., \& Renault, G. (2016). Decomposing Oriented Graphs into Six Locally Irregular Oriented Graphs. Graphs and Combinatorics, 32(5), 1707-1721. https://doi.org/10.1007/s00373-016-1705-z

\section{General rights}

Copyright and moral rights for the publications made accessible in the public portal are retained by the authors and/or other copyright owners and it is a condition of accessing publications that users recognise and abide by the legal requirements associated with these rights.

- Users may download and print one copy of any publication from the public portal for the purpose of private study or research.

- You may not further distribute the material or use it for any profit-making activity or commercial gain

- You may freely distribute the URL identifying the publication in the public portal

If you believe that this document breaches copyright please contact us providing details, and we will remove access to the work immediately and investigate your claim. 


\title{
Decomposing oriented graphs into six locally irregular oriented graphs
}

\author{
Julien Bensmail \\ Department of Applied Mathematics and Computer Science \\ Technical University of Denmark \\ DK-2800 Lyngby, Denmark
}

\author{
Gabriel Renault \\ Univ. Bordeaux, LaBRI, UMR 5800, F-33400 Talence, France \\ CNRS, LaBRI, UMR 5800, F-33400 Talence, France
}

\begin{abstract}
An undirected graph $G$ is locally irregular if every two of its adjacent vertices have distinct degrees. We say that $G$ is decomposable into $k$ locally irregular graphs if there exists a partition $E_{1} \cup E_{2} \cup \ldots \cup E_{k}$ of the edge set $E(G)$ such that each $E_{i}$ induces a locally irregular graph. It was recently conjectured by Baudon et al. that every undirected graph admits a decomposition into at most three locally irregular graphs, except for a well-characterized set of indecomposable graphs. We herein consider an oriented version of this conjecture. Namely, can every oriented graph be decomposed into at most three locally irregular oriented graphs, i.e. whose adjacent vertices have distinct outdegrees? We start by supporting this conjecture by verifying it for several classes of oriented graphs. We then prove a weaker version of this conjecture. Namely, we prove that every oriented graph can be decomposed into at most six locally irregular oriented graphs. We finally prove that even if our conjecture were true, it would remain NP-complete to decide whether an oriented graph is decomposable into at most two locally irregular oriented graphs.
\end{abstract}

Keywords: oriented graph, locally irregular oriented graph, decomposition into locally irregular graphs, complexity

\section{Introduction}

A common class of graphs is the class of regular graphs, which are graphs whose all vertices have the same degree. One could naturally wonder about an antonym 
notion of irregular graphs. In this scope, maybe the most natural definition for an irregular graph could be a graph whose all vertices have distinct degrees. Unfortunately this definition is not suitable for undirected simple graphs as an easy argument shows that such a graph with at least two vertices necessarily has two vertices with the same degree. Indeed, assume $G$ has $n$ vertices and is irregular. Since $G$ is simple, the degree sequence of its vertices is $(0,1, \ldots, n-1)$. But if one vertex of $G$ has degree $n-1$, then all of its vertices have degree at least 1. This contradicts the fact that some vertex of $G$ has degree 0 .

Several studies then aimed at finding ways for introducing irregularity in undirected simple graphs. One first solution, introduced by Chartrand et al. [2], is to turn an undirected simple graph $G$ into an irregular multigraph, i.e. a graph with multiple edges, by multiplying the edges of $G$ (i.e. replacing them with several multiple edges). This led to the introduction of the notion of irregularity strength, where the irregularity strength $s(G)$ of $G$ can be defined as the least integer $s(G) \geq 2$ for which $G$ can be turned into an irregular multigraph by multiplying each of its edges at most $s(G)$ times.

Since the notion of irregularity above does not fit with undirected simple graphs, we may as well consider a weaker notion of irregularity. In connexion with the investigations from [1], which inspired the present work, we here consider graphs that are irregular locally, rather than totally. In particular, by a locally irregular graph, we refer to a graph whose every two adjacent vertices have distinct degrees. An undirected simple graph $G$ may of course not be locally irregular, e.g. if $G$ is regular, so once again we could try to turn $G$ into a locally irregular multigraph (this consideration gave birth to the so-called 1-2-3 Conjecture, see $[6])$.

Another direction for introducing irregularity in $G$ is rather to try to "decompose" $G$ into several locally irregular graphs. More formally, we say that $G$ is decomposable into $k$ locally irregular graphs if there exists a partition $E_{1} \cup E_{2} \cup \ldots \cup E_{k}$ of $E(G)$ such that $G\left[E_{i}\right]$ is locally irregular for every $i \in$ $\{1,2, \ldots, k\}$. Note that finding a decomposition of $G$ into $k$ locally irregular graphs is similar to finding an improper $k$-edge-colouring of $G$ such that each colour class induces a locally irregular graph. Such an edge-colouring is said locally irregular for convenience. As usual, we are interested in the least number of colours used by a locally irregular edge-colouring of $G$. This parameter is called the irregular chromatic index of $G$, denoted $\chi_{\mathrm{irr}}^{\prime}(G)$, and is defined as $\infty$ when $G$ does not admit any locally irregular edge-colouring (note e.g. that the irregular chromatic index of every path or cycle with odd length is not finite). Equivalently, the $\chi_{\mathrm{irr}}^{\prime}(G)$ parameter can be seen as a "measure" of how far from locally irregular $G$ is.

It is conjectured that every undirected "colourable" graph $G$, i.e. whose irregular chromatic index is finite, can be decomposed into at most three locally irregular graphs [1]. This conjecture was verified for several classes of graphs, including trees, complete graphs, and regular graphs with large degree which are, in some sense, the least locally irregular graphs. However, no constant upper bound on the number of necessary colours has been found so far, so no weaker version of the conjecture mentioned above has been proved. 
We here consider this problem in the context of oriented graphs, where the notions of regularity and (local) irregularity are with respect to the outdegree parameter $^{1}$. Namely, an oriented graph $\vec{G}$ is regular (resp. irregular) if all its vertices have the same (resp. distinct) outdegrees. In case every two adjacent vertices of $\vec{G}$ have distinct outdegrees, we say that $\vec{G}$ is locally irregular. The notions of locally irregular arc-colouring and irregular chromatic index of $\vec{G}$ then follow naturally as in the undirected case. Note that, as for the undirected case, our investigations are somehow justified since $\vec{G}$ is irregular if and only if $\vec{G}$ is a transitive tournament. Hence irregular oriented graphs have a very restricted structure. Note further that a single arc is a locally irregular oriented graph, while a single edge is clearly not a locally irregular graph. Therefore, the irregular chromatic index of every oriented graph is defined since a locally irregular $|A(\vec{G})|$-arc-colouring of $\vec{G}$, where $A(\vec{G})$ is the set of arcs of $\vec{G}$, is obtained by assigning one distinct colour to each arc of $\vec{G}$.

Investigations on small oriented graphs suggest that, as for the undirected case, the irregular chromatic index of every oriented graph should be bounded above by 3 .

Conjecture 1. For every oriented graph $\vec{G}$, we have $\chi_{\mathrm{irr}}^{\prime}(\vec{G}) \leq 3$.

This paper is mainly devoted to Conjecture 1. We support this conjecture by showing it to hold for several classes of graphs in Section 2 . We then prove a weaker version of Conjecture 1 in Section 3. Namely, we prove that $\chi_{\text {irr }}^{\prime}(\vec{G}) \leq 6$ holds for every oriented graph $\vec{G}$. We finally turn our concerns on algorithmic aspects in Section 4. In particular, we prove that deciding whether the irregular chromatic index of an oriented graph is at most 2 is an NP-complete problem. In case Conjecture 1 turned out to be true, this would imply that oriented graphs with irregular chromatic index 2 are not easy to recognize, unless $\mathrm{P}=$ NP. Some concluding remarks and open questions are gathered in concluding Section 5.

\section{Some terminology and notation}

Let $\vec{G}$ be an oriented graph, and $\phi$ be a $k$-arc-colouring of $\vec{G}$ for some $k \geq 1$. We denote by $V(\vec{G})$ and $A(\vec{G})$ the vertex and arc sets of $\vec{G}$, respectively. Throughout this paper, the $i$-subgraph of $\vec{G}$ (induced by $\phi$ ) refers to the subgraph of $\vec{G}$ induced by colour $i$ of $\phi$ for any $i \in\{1,2, \ldots, k\}$. Given a vertex $v$ of $\vec{G}$, the $i$-outdegree of $v$ refers to the outdegree of $v$ in the $i$-subgraph. We denote this parameter $d_{\phi, i}^{+}(v)$. We refer the reader to [3] for every other usual notation or terminology.

\footnotetext{
${ }^{1}$ Note that our investigations could have be done with respect to the indegrees instead.
} 


\section{Oriented graphs supporting Conjecture 1}

Throughout this section, we exhibit families of oriented graphs for which Conjecture 1 holds. Namely, we prove Conjecture 1 to hold for oriented graphs whose underlying graph has chromatic number at most 3 , acyclic oriented graphs, and Cartesian products of oriented graphs with irregular chromatic index at most 3 .

\subsection{Oriented graphs whose underlying graph is $k$-colourable}

The underlying graph of an oriented graph $\vec{G}$, denoted und $(\vec{G})$, is the undirected graph obtained from $\vec{G}$ by "replacing" every arc by an edge. A proper $k$ vertex-colouring of an undirected graph $G$ is a partition of $V(G)$ into $k$ parts $V_{1} \cup V_{2} \cup \ldots \cup V_{k}$ such that $V_{i}$ is an independent set for every $i \in\{1,2, \ldots, k\}$. The chromatic number $\chi(G)$ of $G$ is the least number of colours in a proper vertex-colouring of $G$. In the next result, we show that every oriented graph whose underlying graph is $k$-colourable, i.e. admits a proper $k$-vertex-colouring, admits a locally irregular $k$-arc-colouring.

Theorem 2. For every oriented graph $\vec{G}$, we have $\chi_{\text {irr }}^{\prime}(\vec{G}) \leq \chi(\operatorname{und}(\vec{G}))$.

Proof. Without loss of generality, we may assume that und $(\vec{G})$ is connected. Let $\chi(\operatorname{und}(\vec{G}))=k$, and $V_{1} \cup V_{2} \cup \ldots \cup V_{k}$ be a proper $k$-vertex-colouring of und $(\vec{G})$. Consider the $k$-arc-colouring $\phi$ of $\vec{G}$ obtained by colouring $i$ every arc whose tail lies in $V_{i}$ for every $i \in\{1,2, \ldots, k\}$. Now consider every two adjacent vertices $u$ and $v$. By definition of a proper vertex-colouring, we have $u \in V_{i}$ and $v \in V_{j}$ for some $i, j \in\{1,2, \ldots, k\}$ with $i \neq j$. Besides, according to how $\phi$ was obtained, we have $d_{\phi, i}^{+}(u) \geq 0$ and $d_{\phi, j}^{+}(u)=0$, and $d_{\phi, i}^{+}(v)=0$ and $d_{\phi, j}^{+}(v) \geq 0$, while the arc between $u$ and $v$ is coloured either $i$ or $j$. It should be thus clear that $\phi$ is locally irregular.

As a special case of Theorem 2, we get that every oriented graph whose underlying graph is a tree, a bipartite graph, or more generally a 3-colourable graph agrees with Conjecture 1.

Corollary 3. For every oriented graph $\vec{G}$ whose underlying graph is 3-colourable, we have $\chi_{\mathrm{irr}}^{\prime}(\vec{G}) \leq 3$.

\subsection{Acyclic oriented graphs}

An oriented graph is acyclic if it does not admit a circuit as a subgraph. We herein show that every acyclic oriented graph admits a locally irregular 3-arccolouring. The degree bound is best possible, as there exist acyclic oriented graphs with irregular chromatic index 3 (see our results in Section 4).

Theorem 4. For every acyclic oriented graph $\vec{G}$, we have $\chi_{\mathrm{irr}}^{\prime}(\vec{G}) \leq 3$. 
Proof. We actually prove a stronger statement, namely that every acyclic oriented graph $\vec{G}$ admits a locally irregular 3 -arc-colouring in which only two colours are used at each vertex, i.e. the arcs outgoing from every vertex are coloured with at most two colours. The proof is by induction on the order $n$ of $\vec{G}$, i.e. its number of vertices. The claim can be easily verified for small values of $n$, e.g. for $n \leq 3$. Let us thus assume the thesis holds for every oriented graph with order at most $n-1$. Since $\vec{G}$ is acyclic, there has to be a vertex of $\vec{G}$ with indegree 0 . Let $v$ be such a vertex, and denote its neighbours by $u_{1}, u_{2}, \ldots, u_{d}$, i.e. $\overrightarrow{v u_{i}}$ is an arc for every $i \in\{1,2, \ldots, d\}$, where $d=d^{+}(v)$.

Let $\vec{H}=\vec{G}-v$. Since removing vertices from an acyclic graph does not create new circuits, the oriented graph $\vec{H}$ is still acyclic. Besides, it admits a locally irregular 3 -arc-colouring $\phi_{\vec{H}}$ with the restrictions above according to the induction hypothesis. Now put back $v$ and its incident arcs, and try to extend $\phi_{\vec{H}}$, i.e. colour the arcs outgoing from $v$, to a locally irregular 3-arc-colouring $\phi_{\vec{G}}$ of $\vec{G}$ satisfying the conditions above. We show below that such an extension from $\phi_{\vec{H}}$ to $\phi_{\vec{G}}$ necessarily exists.

For this purpose, we first show that such an extension necessarily exists whenever $d \leq 3$ before generalizing our arguments. If $d=1$, then, by our assumptions on $\phi_{\vec{H}}$, at most two colours, say 1 and 2 , are used at $u_{1}$. Then by colouring 3 the arc $\overrightarrow{v u_{1}}$, no conflict may arise and $\phi_{\vec{G}}$ remains locally irregular. Besides, only one colour is used at $v$.

Now, if $d=2$, then start by colouring 1 all arcs outgoing from $v$. If $\phi_{\vec{G}}$ is not locally irregular, then one vertex $u_{i_{1}}$ has 1-outdegree 2 by $\phi_{\vec{H}}$. Now colour 2 all arcs outgoing from $v$. Again, if $\phi_{\vec{G}}$ is not locally irregular, then it means that one vertex $u_{i_{2}}$ has 2 -outdegree 2 by $\phi_{\vec{H}}$. Now colour 3 all arcs outgoing from $v$. If $\phi_{\vec{G}}$ is not locally irregular again, then some vertex $u_{i_{3}}$ has 3 -outdegree 2 . Since $d=2$ and there are at most two colours used at each of the $u_{i}$ 's, it means that we have revealed all the colours used at one of the $u_{i}$ 's. Assume $i_{1}=i_{2}=1$ without loss of generality. Then $u_{1}$ has 1 - and 2-outdegree 2 , while $u_{2}$ has 3 outdegree 2 . Note then that by setting $\phi_{\vec{G}}\left(\overrightarrow{v u_{1}}\right)=1$ and $\phi_{\vec{G}}\left(\overrightarrow{v u_{2}}\right)=3$, the arc-colouring $\phi_{\vec{G}}$ is locally irregular. Since $d=2$, note further that at most two colours are used at $v$, as required.

Finally consider $d=3$. As previously, start by colouring all arcs outgoing from $v$ with a same colour. Again, if $\phi_{\vec{G}}$ is not locally irregular for every of these three extensions of $\phi_{\vec{H}}$, then we get that a vertex $u_{i_{1}}$ has 1-outdegree 3 , a vertex $u_{i_{2}}$ has 2 -outdegree 3 , and one vertex $u_{i_{3}}$ has 3 -outdegree 3 . Now fix $\phi_{\vec{G}}\left(\overrightarrow{v u_{i_{1}}}\right)=1$ (there is no conflict in the 1-subgraph since $u_{i_{1}}$ has 1-outdegree 3 ) and colour all of the remaining arcs outgoing from $v$ with a same colour different from 1. Again, if $\phi_{\vec{G}}$ is never locally irregular, then we get that some vertex $u_{i_{4}}$ has 2-outdegree 2, and some vertex $u_{i_{5}}$ has 3-outdegree 2. Similarly, if $\phi_{\vec{G}}$ is not locally irregular when assigning $\overrightarrow{v u_{i_{2}}}$ colour 2 (again, there is not conflict in the 2-subgraph since $u_{i_{2}}$ has 2-outdegree 3 ) and all of the other arcs outgoing from $v$ colour 1 , then we get that some vertex $u_{i_{6}}$ has 1-outdegree 2. At this point, all of the colours of the arcs outgoing from the $u_{i}$ 's are revealed. Since 
it was revealed that colour 1 is used at $u_{i_{6}}$, either colour 2 or 3 is not used at $v_{i_{6}}$. Assume this colour is 2 without loss of generality. Now just assign colour 2 to $\overrightarrow{v u_{i_{6}}}$, and colour 1 to all of the other arcs outgoing from $v$. Then $v$ and $u_{i_{6}}$ are adjacent in the 2-subgraph but have distinct 2-outdegrees, namely 1 and 0 , respectively, while $v$ and its other two neighbours are adjacent in the 1subgraph and have distinct 1-outdegrees since $v$ has 1-outdegree 2 and only $u_{i_{6}}$ was revealed to have 1-outdegree 2 . It then follows that $\phi_{\vec{G}}$ is locally irregular. Note further that at most two colours are used to colour the arcs outgoing from $v$ at every moment of the procedure.

We now generalize our arguments for every $d \geq 4$. The colouring scheme we use below is quite similar to the one used so far. If, at some step, the resulting arc-colouring $\phi_{\vec{G}}$ is locally irregular, then we are done. Suppose this never occurs. We start by colouring with only one colour all arcs outgoing from $v$ (Step 1). Since $\phi_{\vec{G}}$ is never locally irregular, it means that some vertex $u_{i_{1}}$ has 1-outdegree $d$, some vertex $u_{i_{2}}$ has 2 -outdegree $d$, and some vertex $u_{i_{3}}$ has 3-outdegree $d$. Next, we try to extend $\phi_{\vec{H}}$ to $\phi_{\vec{G}}$ by colouring with some colour $\alpha$ one arc outgoing from $v$ whose head was shown to have $\alpha$-outdegree strictly more than 1 in earlier steps of the process (i.e. $u_{i_{1}}, u_{i_{2}}$ or $u_{i_{3}}$ ), and then colouring all of the other arcs outgoing from $v$ with a colour different from $\alpha$ (Step 2.a). Again, if $\phi_{\vec{G}}$ is not locally irregular for every of these attempts, then we reveal that some vertex $u_{i_{4}}$ has 1 -outdegree $d-1$, some vertex $u_{i_{5}}$ has 2 -outdegree $d-1$, and some vertex $u_{i_{6}}$ has 3 -outdegree $d-1$. Once the vertices $u_{i_{4}}, u_{i_{5}}$ and $u_{i_{6}}$ are revealed, we can reveal additional 1-, 2- and 3-outdegrees as follows. Since $u_{i_{4}}$ has 1 -outdegree $d-1$, it means that a colour different from 1 , say 2 , is not used at $u_{i_{4}}$. Then colour 2 the arc $\overrightarrow{v u_{i_{4}}}$, and 1 all of the other arcs outgoing from $v$. Then we reveal that a vertex $u_{i_{7}}$ different from $u_{i_{1}}$ and $u_{i_{4}}$ has 1-outdegree $d-1$. Repeating the same strategy with $u_{i_{5}}$ and $u_{i_{6}}$ (Step 2.b), we reveal also that two vertices $u_{i_{8}}$ (different from $u_{i_{2}}$ and $u_{i_{5}}$ ) and $u_{i_{9}}$ (different from $u_{i_{3}}$ and $u_{i_{6}}$ ) have 2 - and 3-outdegree $d-1$, respectively.

Repeat the same strategy as many times as necessary until $\phi_{\vec{G}}$ is locally irregular, or all of the 1-, 2- and 3-outdegrees of the $u_{i}$ 's are revealed. More precisely, for every $j=3,4, \ldots,\left\lceil\frac{d}{2}\right\rceil$ taken consecutively, colour with some colour $\alpha$ exactly $j-1$ of the arcs outgoing from $v$ whose heads were shown to have $\alpha$-outdegree strictly more than $j-1$ in earlier steps, and colour the remaining $d-j+1$ arcs with some different colour $\beta \neq \alpha$ (Step j.a). At Step j.a, we reveal that some vertex $u_{i_{3+6(j-2)+1}}$ has 1 -outdegree $d-j+1$, some vertex $u_{i_{3+6(j-2)+2}}$ has 2 -outdegree $d-j+1$, and some vertex $u_{i_{3+6(j-2)+3}}$ has 3 outdegree $d-j+1$. Repeating Step j.a but with "forcing" $u_{i_{3+6(j-2)+1}}$ to be one of the $j-1$ arcs coloured with some colour not appearing at it, and then similarly for $u_{i_{3+6(j-2)+2}}$ and $u_{i_{3+6(j-2)+3}}$ (Step j.b), we reveal that three other vertices, $u_{i_{3+6(j-2)+4}}, u_{i_{3+6(j-2)+5}}$ and $u_{i_{3+6(j-1)}}$, have 1 -, 2 - and 3 -outdegree $d-j+1$, respectively. We refer to Steps j.a and j.b as Step j.

Hence, at each Step $\mathrm{j}$, we reveal that two of the $u_{i}$ 's have 1-outdegree $d-$ $j+1$, two of the $u_{i}$ 's have 2-outdegree $d-j+1$ and two of the $u_{i}$ 's have 3 outdegree $d-j+1$ (except for Step 1 where only one outdegree of each colour is revealed). Since $d \geq 4$ and there are only two colours used at each vertex 
$u_{i}$ according to the assumption on $\phi_{\vec{H}}$, and hence at most $2 d$ outdegrees to be revealed, it should be clear that all of the 1-, 2- and 3-outdegrees of the $u_{i}$ 's are revealed once $j$ reaches $\left\lceil\frac{d}{2}\right\rceil$. Besides, every 1-, 2- or 3-outdegree is revealed to be strictly more than $\left\lceil\frac{d}{2}\right\rceil$ (except when $d=5$, see below). One can then obtain the locally irregular 3 -arc-colouring $\phi_{\vec{G}}$ by assigning colour 1 to $\left\lceil\frac{d}{2}\right\rceil \operatorname{arcs}$ outgoing from $v$ whose head were shown to have 1-outdegree strictly more than $\left\lceil\frac{d}{2}\right\rceil$, and colour 2 to the remaining arcs (there are $\left\lfloor\frac{d}{2}\right\rfloor$ of them). Under this colouring, the vertex $v$ has 1 - and 2-outdegree $\left\lceil\frac{d}{2}\right\rceil$ and $\left\lfloor\frac{d}{2}\right\rfloor$, respectively, while its neighbours have 1 - and 2-outdegree 0 or strictly greater than these values in the 1- and 2-subgraphs, respectively (when $d=5$, one of the $u_{i}$ 's, say $u_{1}$, is revealed to have 1-outdegree 3 - in this special case, force $\overrightarrow{v u_{1}}$ to be coloured 2 . For every other value of $d$, the revealed outdegrees are strictly greater than $\left\lceil\frac{d}{2}\right\rceil$ ). Besides, only two colours are used at $v$. This ends up the proof.

It is worth noting that the stronger statement proved in the proof of Theorem 4 is crucial for our colouring scheme. Indeed, assume e.g. that $d=1$, that three colours are allowed at each vertex, and that $u_{1}$ has 1-, 2- and 3-outdegree exactly 1 by $\phi_{\vec{H}}$. In such a situation, we clearly cannot extend $\phi_{\vec{H}}$ to $\phi_{\vec{G}}$.

\subsection{Cartesian products of oriented graphs with irregular chromatic index at most 3}

We herein investigate a last family of oriented graphs, namely Cartesian products of oriented graphs with irregular chromatic index at most 3 (the reader is referred to [5] for details on this construction). The main interest for focusing on this operation is that it provides numerous more examples of oriented graphs verifying Conjecture 1, assuming that we are provided with oriented graphs agreeing with Conjecture 1 themselves.

Theorem 5. Let $\vec{G}$ and $\vec{H}$ be two oriented graphs such that $\chi_{\mathrm{irr}}^{\prime}(\vec{G}) \leq k$ and $\chi_{\text {irr }}^{\prime}(\vec{H}) \leq \ell$. Then we have $\chi_{\text {irr }}^{\prime}(\vec{G} \square \vec{H}) \leq \max \{k, \ell\}$.

Proof. Let $\phi_{\vec{G}}$ and $\phi_{\vec{H}}$ be locally irregular $k$ - and $\ell$-arc-colourings of $\vec{G}$ and $\vec{H}$, respectively. Now let $\phi \vec{G} \square \vec{H}$ be the $\max \{k, \ell\}$-arc-colouring of $\vec{G} \square \vec{H}$ obtained from $\phi_{\vec{G}}$ and $\phi_{\vec{H}}$ as follows:

$$
\phi_{\vec{G} \square \vec{H}}\left(\overrightarrow{\left(u_{1}, v_{1}\right)\left(u_{2}, v_{2}\right)}\right)=\left\{\begin{array}{l}
\phi_{H}\left(\overrightarrow{v_{1} v_{2}}\right) \text { if } u_{1}=u_{2}, \\
\phi_{G}\left(\overrightarrow{u_{1} u_{2}}\right) \text { otherwise. }
\end{array}\right.
$$

Note that $d_{\phi_{\vec{G} \square \vec{H}}, i}^{+}\left(\left(u_{1}, v_{1}\right)\right)=d_{\phi_{\vec{G}}, i}^{+}\left(u_{1}\right)+d_{\phi_{\vec{H}}, i}^{+}\left(v_{1}\right)$ for every colour $i \in\{1,2$, $\ldots, \max \{k, \ell\}\}$. Assume $\overrightarrow{\left(u_{1}, v_{1}\right)\left(u_{2}, v_{2}\right)}$ is an arc of $\vec{G} \square \vec{H}$ with $\phi_{\vec{G} \square \vec{H}}\left(\overrightarrow{\left(u_{1}, v_{1}\right)\left(u_{2}, v_{2}\right)}\right)=$ $i$. By definition, we have either $u_{1}=u_{2}$ or $v_{1}=v_{2}$. Suppose $u_{1}=u_{2}$ without loss of generality. Then we have $d_{\phi_{\vec{G}}, i}^{+}\left(u_{1}\right)=d_{\phi_{\vec{G}}, i}^{+}\left(u_{2}\right)$, and, because $\phi_{\vec{H}}$ is locally irregular, also $d_{\phi_{\vec{H}}, i}^{+}\left(v_{1}\right) \neq d_{\phi_{\vec{H}}, i}^{+}\left(v_{2}\right)$. It then follows that 
$d_{\phi_{\vec{G} \square \vec{H}}, i}^{+}\left(\left(u_{1}, v_{1}\right)\right) \neq d_{\phi_{\vec{G} \square \vec{H}}, i}^{+}\left(\left(u_{2}, v_{2}\right)\right)$. Repeating the same argument for every arc of $\vec{G} \square \vec{H}$, we get that $\phi_{\vec{G}} \square \vec{H}$ is locally irregular.

Regarding Conjecture 1, we get the following.

Corollary 6. For every two oriented graphs $\vec{G}$ and $\vec{H}$ such that $\chi_{\mathrm{irr}}^{\prime}(\vec{G}), \chi_{\mathrm{irr}}^{\prime}(\vec{H}) \leq$ 3, we have $\chi_{\text {irr }}^{\prime}(\vec{G} \square \vec{H}) \leq 3$.

\section{Decomposing oriented graphs into at most six locally irregular oriented graphs}

In this section we show, in Theorem 8 below, that every oriented graph has irregular chromatic index at most 6 . For this purpose, we first introduce the following observation stating that if an oriented graph $\vec{G}$ can be "decomposed" into arc-disjoint subgraphs $\overrightarrow{G_{1}}, \overrightarrow{G_{2}}, \ldots, \overrightarrow{G_{k}}$, then a locally irregular arc-colouring of $\vec{G}$ can be obtained by considering independent locally irregular arc-colourings of $\overrightarrow{G_{1}}, \overrightarrow{G_{2}}, \ldots, \overrightarrow{G_{k}}$.

Observation 7. Let $\vec{G}$ be an oriented graph whose arc set $A(\vec{G})$ can be partitioned into $k$ parts $A_{1} \cup A_{2} \cup \ldots \cup A_{k}$ such that $\chi_{\mathrm{irr}}^{\prime}\left(\vec{G}\left[A_{1}\right]\right) \leq x_{1}, \chi_{\mathrm{irr}}^{\prime}\left(\vec{G}\left[A_{2}\right]\right) \leq$ $x_{2}, \ldots, \chi_{\mathrm{irr}}^{\prime}\left(\vec{G}\left[A_{k}\right]\right) \leq x_{k}$ for some values of $x_{1}, x_{2}, \ldots, x_{k}$. Then $\chi_{\mathrm{irr}}^{\prime}(\vec{G}) \leq$ $\sum_{i=1}^{k} x_{i}$.

Proof. Let $\phi_{1}, \phi_{2}, \ldots, \phi_{k}$ be locally irregular $x_{1^{-}}, x_{2^{-}}, \ldots, x_{k^{-}}$-arc-colourings of $\vec{G}\left[A_{1}\right], \vec{G}\left[A_{2}\right], \ldots, \vec{G}\left[A_{k}\right]$, respectively, and denote by $\phi$ the $\left(\sum_{i=1}^{k} x_{i}\right)$-arccolouring of $\vec{G}$ defined as

$$
\phi(\vec{a})=\left(\phi_{i}(\vec{a}), i\right) \text { for every } \vec{a} \in A(\vec{G}) \text { such that } \vec{a} \in A_{i} .
$$

By the partition of $A(\vec{G})$, every arc of $\vec{G}$ receives a colour by $\phi$, and $\phi$ uses $\sum_{i=1}^{k} x_{i}$ colours. Besides, the subgraph of $\vec{G}$ induced by colour $(j, i)$ is nothing but the subgraph of $\vec{G}\left[A_{i}\right]$ induced by colour $j$ of $\phi_{i}$, which is locally irregular by the definition of $\phi_{i}$. All subgraphs of $\vec{G}$ induced by $\phi$ are then locally irregular as required.

Observation 7 provides an easy upper bound on the irregular chromatic index of every oriented graph which may be decomposed into arc-disjoint subgraphs with bounded irregular chromatic index. In particular, by showing below that every oriented graph can be arc-partitioned into two acyclic oriented graphs (which have irregular chromatic index at most 3, see Theorem 4), we directly get that every oriented graph has irregular chromatic index at most 6 .

Lemma 8. The arc set of every oriented graph $\vec{G}$ can be partitioned into two parts $A_{1} \cup A_{2}$ such that $\vec{G}\left[A_{1}\right]$ and $\vec{G}\left[A_{2}\right]$ are acyclic. 
Proof. Let $v_{1}, v_{2}, \ldots, v_{n}$ denote the vertices of $\vec{G}$ following an arbitrary order. Now consider every arc $\overrightarrow{v_{i} v_{j}}$ of $\vec{G}$, and

$$
\left\{\begin{array}{l}
\text { add } \overrightarrow{v_{i} v_{j}} \text { to } A_{1} \text { if } i<j, \\
\text { add } \overrightarrow{v_{i} v_{j}} \text { to } A_{2} \text { otherwise. }
\end{array}\right.
$$

Then observe that if $\overrightarrow{v_{i_{1}} v_{i_{2}} \ldots v_{i_{k}} v_{i_{1}}}$, with $i_{1}<i_{2}<\ldots<i_{k}$, were a circuit of $\vec{G}\left[A_{1}\right]$, then we would have both $i_{1}<i_{k}$ and $i_{k}<i_{1}$, a contradiction. A similar contradiction can be deduced from any circuit of $\vec{G}\left[A_{2}\right]$.

Theorem 9. For every oriented graph $\vec{G}$, we have $\chi_{\text {irr }}^{\prime}(\vec{G}) \leq 6$.

Proof. According to Lemma 3, there exists a partition $A_{1} \cup A_{2}$ of $A(\vec{G})$ such that $\vec{G}\left[A_{1}\right]$ and $\vec{G}\left[A_{2}\right]$ are acyclic. Since every acyclic oriented graph has irregular chromatic index at most 3 according to Theorem 4 , the thesis follows directly from Observation 7.

\section{Algorithmic complexity}

In this section, we deal with the algorithmic complexity of the following decision problem.

LoCAlly IrRegular $k$-ArC-COLOURING

Instance: An oriented graph $\vec{G}$.

Question: Do we have $\chi_{\text {irr }}^{\prime}(\vec{G}) \leq k$ ?

Since checking whether $\vec{G}$ is locally irregular can be done in quadratic time, the problem Locally Irregular 1-Arc-Colouring is in P. In case Conjecture 1 turned out to be true, note that every problem Locally IrREgular $k$-Arc-Colouring with $k \geq 3$ would be in P. At the moment, by Theorem 8 we only get that LOCALLY IRREgular $k$-ARC-COLOURING is in P for every $k \geq 6$. On contrary, if LOCALly IrRegular $k$-ArC-Colouring were shown to be NP-complete for some $k \in\{3,4,5\}$, then one would disprove Conjecture 1 .

In the light of the previous explanations, only LOCALLY IRREGULAR 2-ARCColouring is of interest at the moment. In Theorem 9 below, we prove this problem to be NP-complete, even when restricted to acyclic oriented graphs. This result implies that, even if Conjecture 1 turned out to be true, no good characterization of oriented graphs with irregular chromatic index at most 2 can exist, unless $\mathrm{P}=\mathrm{NP}$.

To prove Theorem 9, we first need to introduce some forcing gadgets, i.e. some oriented graphs which will be used in our reduction to "force" the propagation of a locally irregular 2-arc-colouring within an oriented graph.

The 2-fiber gadget, denoted $\vec{F}_{2}$, is depicted in Fig. 1. We refer to the arcs $\overrightarrow{v_{1}^{\prime} v_{1}}, \overrightarrow{v_{2}^{\prime} v_{2}}, . ., \overrightarrow{v_{x}^{\prime} v_{x}}$ as the outputs of $\overrightarrow{F_{2}}$. Every output $\overrightarrow{v_{i}^{\prime} v_{i}}$ with $i$ odd is referred to as an odd output, or as an even output otherwise. Note that $\overrightarrow{F_{2}}$ is 


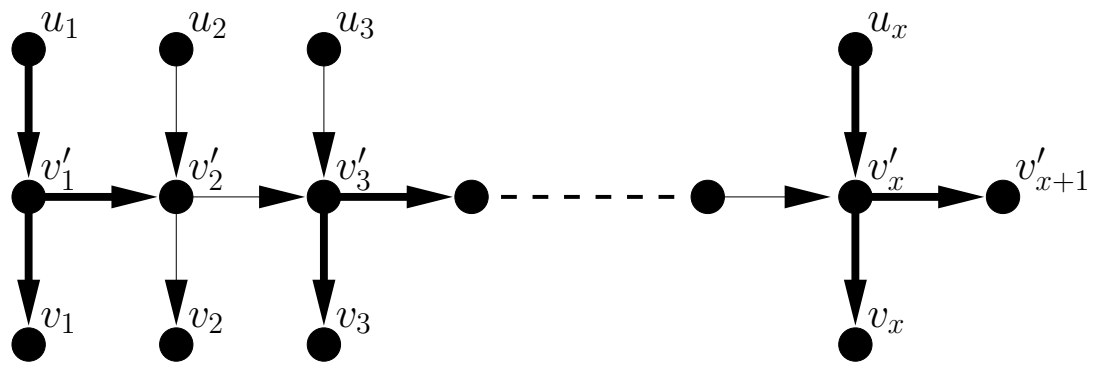

Figure 1: The 2-fiber gadget $\overrightarrow{F_{2}}$, and a locally irregular 2-arc-colouring of $\overrightarrow{F_{2}}$ (thick (resp. thin) arcs are arcs coloured 1 (resp. 2)).

actually made of a same small pattern repeated consecutively from left to right. The dashed section of $\overrightarrow{F_{2}}$ means that this pattern can be repeated an arbitrary number of times, i.e. $x$ can be arbitrarily large, so that $\vec{F}_{2}$ has arbitrarily many outputs, which are either even or odd, alternatively. This gadget has the following colouring property.

Lemma 10. In every locally irregular 2-arc-colouring $\phi$ of $\overrightarrow{F_{2}}$, all of the even outputs of $\overrightarrow{F_{2}}$ have the same colour, while all of the odd outputs have the second colour. Besides, for every output $\overrightarrow{v_{i}^{\prime} v_{i}}$ of $\overrightarrow{F_{2}}$, the vertex $v_{i}^{\prime}$ has outdegree 2 in the $\phi\left(\overrightarrow{v_{i}^{\prime} v_{i}}\right)$-subgraph.

Proof. Note that for every $i \in\{1,2, \ldots, x\}$, the vertex $u_{i}$ has $\alpha$-outdegree 1 by $\phi$ for some $\alpha \in\{1,2\}$ and is adjacent to $v_{i}^{\prime}$ in the $\alpha$-subgraph. For this reason, the two arcs $\overrightarrow{v_{i}^{\prime} v_{i}}$ and $\overrightarrow{v_{i}^{\prime} v_{i+1}^{\prime}}$ cannot have distinct colours by $\phi$ since otherwise $v_{i}^{\prime}$ would have $\alpha$-outdegree 1 too.

Hence, all of the arcs outgoing from $v_{i}^{\prime}$ have the same colour. Suppose e.g. that all of the arcs outgoing from $v_{1}^{\prime}$ are coloured 1 . Then $v_{1}^{\prime}$ has 1-outdegree 2, and $v_{1}^{\prime}$ and $v_{2}^{\prime}$ are adjacent in the 1-subgraph. For these reasons, all of the arcs outgoing from $v_{2}^{\prime}$ cannot be coloured 1 since otherwise $v_{2}^{\prime}$ would have 1outdegree 2 too. Then all of the arcs outgoing from $v_{2}^{\prime}$ are coloured 2 by $\phi$. Repeating the same argument from the left to the right of $\vec{F}_{2}$, we get that $v_{i}^{\prime}$ has 1-outdegree 2 for every odd $i$, while $v_{i}^{\prime}$ has 2 -outdegree 2 otherwise, i.e. when $i$ is even. So every two consecutive outputs of $\overrightarrow{F_{2}}$ have distinct colours.

We now generalize the notion of $k$-fiber gadget for every $k \geq 3$. Consider any value of $k$ such that every $i$-fiber gadget has been defined for every $i \in$ $\{2,3, \ldots, k-1\}$. Start from a directed path $\overrightarrow{v_{1}^{\prime} v_{2}^{\prime} \ldots v_{x}^{\prime} v_{x+1}^{\prime}}$ for some arbitrary value of $x$. For every $v_{i}^{\prime}$ with $i \in\{1,2, \ldots, x\}$, add arcs from $v_{i}^{\prime}$ towards $k-1$ new vertices with outdegree 0 . Call $v_{i}$ one such resulting vertex. Finally, identify $v_{i}^{\prime}$ with the heads of one distinct even output and one distinct odd output of each of $\overrightarrow{F_{2}}, \overrightarrow{F_{3}}, \ldots, \overrightarrow{F_{k-1}}$. Refer to Fig. 2 for an illustration of the 3 -fiber gadget $\overrightarrow{F_{3}}$. Similarly as for $\overrightarrow{F_{2}}$, we refer to the arcs $\overrightarrow{v_{i}^{\prime} v_{i}}$ of $\overrightarrow{F_{k}}$ as its outputs, making again 


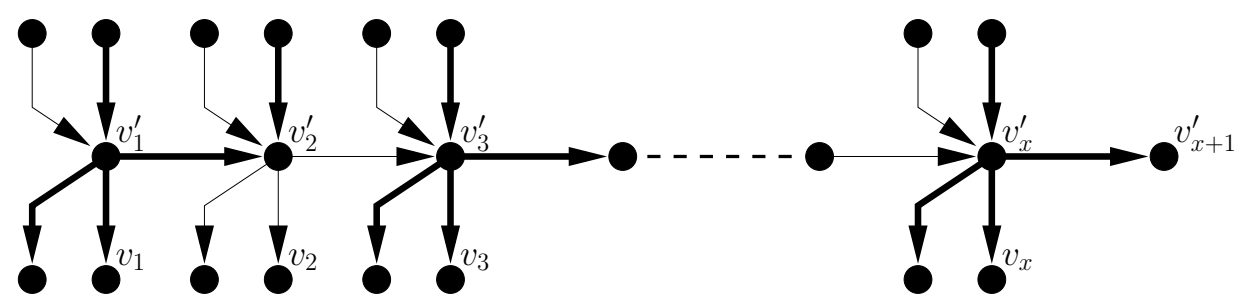

Figure 2: The 3-fiber gadget $\overrightarrow{F_{3}}$, where the top-most arcs are outputs of $\overrightarrow{F_{2}}$, and a locally irregular 2-arc-colouring of $\overrightarrow{F_{3}}$ (thick (resp. thin) arcs are arcs coloured 1 (resp. 2)).

the distinction between even and odd outputs. The generalized fiber gadgets share a similar colouring property as $\overrightarrow{F_{2}}$.

Lemma 11. In every locally irregular 2-arc-colouring $\phi$ of $\overrightarrow{F_{k}}$, all of the even outputs of $\overrightarrow{F_{k}}$ have the same colour, while all of the odd outputs have the second colour. Besides, for every output $\overrightarrow{v_{i}^{\prime} v_{i}}$ of $\overrightarrow{F_{k}}$, the vertex $v_{i}^{\prime}$ has outdegree $k$ in the $\phi\left(\overrightarrow{v_{i}^{\prime} v_{i}}\right)$-subgraph.

Proof. The proof is similar to the one of Lemma 4. Consider every vertex $v_{i}^{\prime}$ of $\overrightarrow{F_{k}}$. Since the heads of one even output and one odd output of $\vec{F}_{j}$ are identified with $v_{i}^{\prime}$ for every $j \in\{2,3, \ldots, k-1\}$, for every such $j$ there are two vertices $w_{j, 1}$ and $w_{j, 2}$ neighbouring $v_{i}^{\prime}$ such that:

- $w_{j, 1}$ and $v_{i}^{\prime}$ are adjacent in the 1-subgraph induced by $\phi$,

- $w_{j, 2}$ and $v_{i}^{\prime}$ are adjacent in the 2-subgraph induced by $\phi$,

- $w_{j, 1}$ has 1-outdegree $j$ and $w_{j, 2}$ has 2-outdegree $j$.

Since this observation holds for every $j \in\{2,3, \ldots, k-1\}$, note that all the arcs outgoing from $v_{i}^{\prime}$ must have the same colour by $\phi$ since otherwise $v_{i}^{\prime}$ would have the same outdegree as one of its neighbours in either the 1- or 2-subgraph. Assume all the arcs outgoing from $v_{1}^{\prime}$ have colour 1 by $\phi$ without loss of generality. Then all arcs outgoing from $v_{2}^{\prime}$ cannot all be coloured 1 since otherwise $v_{1}^{\prime}$ and $v_{2}^{\prime}$ would be adjacent vertices with outdegree $k$ in the 1-subgraph. Then all arcs outgoing from $v_{2}^{\prime}$ have colour 2 by $\phi$. Again, by repeating this argument from left to right, similarly as in the proof of Lemma 4, we get that the colours of the outputs of $\overrightarrow{F_{k}}$ alternate between 1 and 2 , and that the tail of each output has $\alpha$-outdegree $k$, where $\alpha$ is the colour of this output by $\phi$.

The generalized fiber gadgets described above are actually not all necessary to prove our main result, but using these we can "generate" vertices with arbitrarily large outdegree in either the 1- or 2-subgraph induced by a locally irregular 2-arc-colouring of some oriented graph. Used conveniently (note in 
particular that if we identify the heads of one even output and one odd output of, say, $\overrightarrow{F_{2}}$, with a vertex $v$, then $v$ cannot have outdegree 2 in the 1 - and 2 -subgraphs by some locally irregular 2-arc-colouring), one can construct arbitrarily many oriented graphs with various structures and which have irregular chromatic index 3 . This should convince the reader that even if Conjecture 1 turned out to be true, oriented graphs with irregular chromatic index 2 do not have a predictable structure.

We are now ready to prove the main result of this section.

Theorem 12. Locally Irregular 2-Arc-Colouring is NP-complete.

Proof. Clearly Locally Irregular 2-ArC-Colouring is in NP since, given a 2-arc-colouring of $\vec{G}$, one can easily check whether the two subgraphs of $\vec{G}$ it induces are locally irregular (this property can be checked in quadratic time).

We now prove that LocAlly IrRegular 2-ArC-COLOURING is NP-hard, and thus NP-complete since it is also in NP, by reduction from the following well-known NP-hard problem [4].

Not-All-Equal 3-Satisfiability

Instance: A $3 \mathrm{CNF}$ formula $F$ over variables $x_{1}, x_{2}, \ldots, x_{n}$ and clauses $C_{1}, C_{2}, \ldots, C_{m}$. Question: Is $F$ nae-satisfiable, i.e. does there exist a truth assignment to the variables of $F$ such that every clause of $F$ has at least one true and one false literal?

Not-All-Equal 3-SATisfiability is notoriously hard, even in its monotone form, i.e. when restricted to instances with no negated variable [4]. We hence suppose throughout this proof that every of its instances, i.e. every formula $F$, has no negated variable.

From $F$, we construct an oriented graph $\overrightarrow{G_{F}}$ such that

$$
\begin{gathered}
F \text { is nae-satisfiable } \\
\Leftrightarrow \\
\overrightarrow{G_{F}} \text { admits a locally irregular 2-arc-colouring } \phi_{F} .
\end{gathered}
$$

We design $\overrightarrow{G_{F}}$ in such a way that the propagation of $\phi_{F}$ along $\overrightarrow{G_{F}}$ is representative of the constraints attached to Not-AlL-EQUAL 3-SATisfiabiLity, i.e. of the consequences on $F$ of setting such or such variable of $F$ to true. This is typically done by designing gadgets with specific colouring properties. Throughout this proof, colour 1 of $\phi_{F}$ must be thought of as the truth value true, while colour 2 represents the truth value false of a truth assignment to the variables of $F$ (one could actually switch these two equivalences as we are dealing with Not-AlL-Equal 3-SATisfiability).

The first requirement of Not-AlL-EQUAL 3-SATisfiability we have to "translate" is that every clause of $F$ is considered satisfied if and only if it has at least one true and one false variable. This is done by "transforming" every clause $C_{j}=\left(x_{i_{1}} \vee x_{i_{2}} \vee x_{i_{3}}\right)$ into some clause gadget $\overrightarrow{G_{F}}\left(C_{j}\right)$ in $\overrightarrow{G_{F}}$ with three special arcs $\overrightarrow{a_{1}}, \overrightarrow{a_{2}}$ and $\overrightarrow{a_{3}}$ representing the variables of $C_{j}$, and such that all 


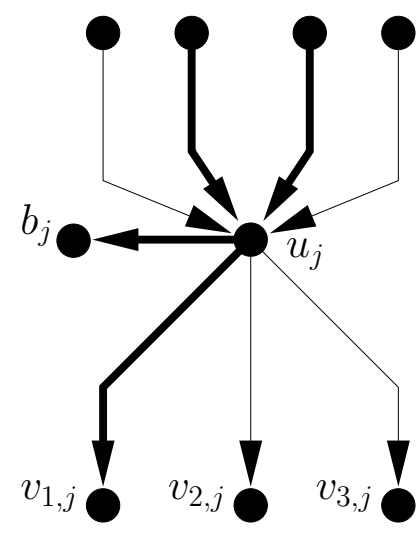

Figure 3: The clause gadget $\overrightarrow{G_{F}}\left(C_{j}\right)$, where the top-most arcs are outputs of the 3 - and 4-fiber gadgets $\vec{F}_{3}$ and $\overrightarrow{F_{4}}$, and a locally irregular 2-arc-colouring of $\overrightarrow{G_{F}}\left(C_{j}\right)$ (thick (resp. thin) arcs are arcs coloured 1 (resp. 2)).

of these three arcs cannot have the same colour by $\phi_{F}$. Assuming that, say, $\phi_{F}\left(\overrightarrow{a_{1}}\right)=1$ (resp. $\phi_{F}\left(\overrightarrow{a_{1}}\right)=2$ ) simulates the fact that $x_{i_{1}}$ supplies $C_{j}$ with value true (resp. false), the requirement above then follows naturally from the colouring property of $\overrightarrow{G_{F}}\left(C_{j}\right)$.

Consider then every clause $C_{j}=\left(x_{i_{1}} \vee x_{i_{2}} \vee x_{i_{3}}\right)$, whose some variables may be the same. The clause gadget $\overrightarrow{G_{F}}\left(C_{j}\right)$, associated with $C_{j}$, is obtained as follows (see Fig. 3). Add five vertices $u_{j}, v_{1, j}, v_{2, j}, v_{3, j}$ and $b_{j}$ to $\overrightarrow{G_{F}}$, as well as all arcs from $u_{j}$ towards every vertex in $\left\{v_{1, j}, v_{2, j}, v_{3, j}, b_{j}\right\}$. Now identify $u_{j}$ with the heads of one even output and one odd output of each of $\vec{F}_{3}$ and $\overrightarrow{F_{4}}$, where $\overrightarrow{F_{3}}$ and $\overrightarrow{F_{4}}$ are the 3 - and 4 -fiber gadgets. The arcs $\overrightarrow{a_{1}}, \overrightarrow{a_{2}}$ and $\overrightarrow{a_{3}}$ mentioned in the explanations above actually refer to $\overrightarrow{u_{j} v_{1, j}}, \overrightarrow{u_{j} v_{2, j}}$ and $\overrightarrow{u_{j} v_{3, j}}$. Besides, one has to think of every vertex $v_{i, j}$ as a vertex associated with the $i$ th variable of $C_{j}$. We show that $\overrightarrow{G_{F}}\left(C_{j}\right)$ cannot have all of its $\operatorname{arcs} \overrightarrow{u_{j} v_{1, j}}, \overrightarrow{u_{j} v_{2, j}}$ and $\overrightarrow{u_{j} v_{3, j}}$ having the same colour by $\phi_{F}$, as required.

Claim 1. Let $C_{j}$ be a clause of $F$, with $j \in\{1,2, \ldots, m\}$. Then one arc of $\overrightarrow{u_{j} v_{1, j}}, \overrightarrow{u_{j} v_{2, j}}$ and $\overrightarrow{u_{j} v_{3, j}}$ has some colour by $\phi_{F}$, while the other two arcs have the second colour.

Proof. The claim follows from the facts that $u_{j}$ has outdegree 4 and is adjacent to vertices with outdegree 3 and 4 in both the 1- and 2-subgraphs induced by $\phi_{F}$, namely the tails of some outputs of $\overrightarrow{F_{3}}$ and $\overrightarrow{F_{4}}$ whose heads were identified with $u_{j}$.

The second requirement of Not-AlL-Equal 3-SATiSfiABILITY we have to model is that, by a truth assignment of the variables of $F$, a variable provides 


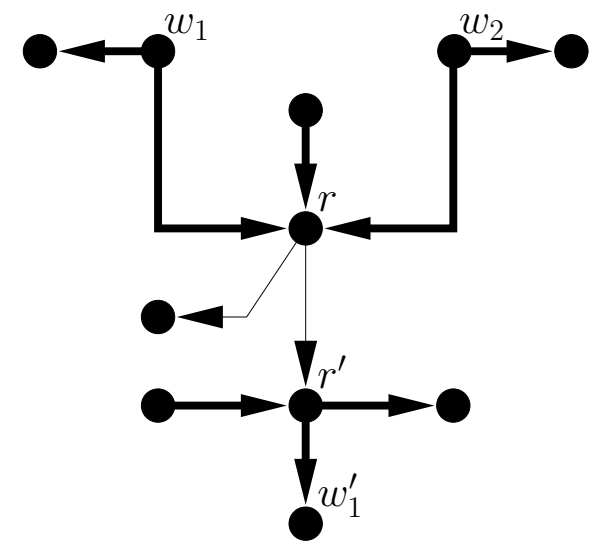

Figure 4: The collecting gadget $\overrightarrow{G^{\curlyvee}}$, and a locally irregular 2-arc-colouring of $\overrightarrow{G^{\curlyvee}}$ (thick (resp. thin) arcs are arcs coloured 1 (resp. 2)).

the same truth value to every clause it appears in. At the moment, this requirement is not met as $\phi_{F}$ may be locally irregular but with, say, $\phi_{F}\left(\overrightarrow{u_{j} v_{i, j}}\right)=1$ and $\phi_{F}\left(\overrightarrow{u_{j^{\prime}} v_{i^{\prime}, j^{\prime}}}\right)=2$ with the $i$ th variable of $C_{j}$ being identical to the $i^{\prime}$ th variable of $C_{j^{\prime}}$, say $x_{\ell}$. Following our analogy above, this would simulates that $x_{\ell}$ belongs to both of the clauses $C_{j}$ and $C_{j^{\prime}}$, but $x_{\ell}$ provides true to $C_{j}$ and false to $C_{j^{\prime}}$ by a truth assignment, which is impossible. Hence, we have to check somehow whether all the $\operatorname{arcs} \overrightarrow{u_{j_{1}} v_{i_{1}, j_{1}}}, \overrightarrow{u_{j_{2}} v_{i_{2}, j_{2}}}, \ldots, \overrightarrow{u_{j_{n_{i}}} v_{i_{n_{i}}, j_{n_{i}}}}$, representing the membership of $x_{\ell}$ to the clauses $C_{j_{1}}, C_{j_{2}}, \ldots, C_{j_{n_{i}}}$ of $F$ that contain $x_{\ell}$, have the same colour by $\phi_{F}$.

This is done by using the collecting gadget $\overrightarrow{G^{\curlyvee}}$ depicted in Fig. 4. The arcs $\overrightarrow{w_{1} r}$ and $\overrightarrow{w_{2} r}$ are called the inputs of $\overrightarrow{G^{r}}$, while $\overrightarrow{r^{\prime} w_{1}^{\prime}}$ is its output. Note that $w_{1}, w_{2}$ and $r^{\prime}$ have outdegree 2 . This gadget $\overrightarrow{G^{\curlyvee}}$ has the following colouring property.

Claim 2. Let $\phi$ be a locally irregular 2-arc-colouring of $\overrightarrow{G^{r}}$ such that the two arcs outgoing from $w_{1}$ have the same colour, and the two arcs outgoing from $w_{2}$ have the same colour. Then $\phi\left(\overrightarrow{w_{1} r}\right)=\phi\left(\overrightarrow{w_{2} r}\right)=\phi\left(\overrightarrow{r^{\prime} w_{1}^{\prime}}\right)$.

Proof. Assume $\phi\left(\overrightarrow{w_{1} r}\right)=1$ and $\phi\left(\overrightarrow{w_{2} r}\right)=2$ without loss of generality. In particular, note that $w_{1}$ and $r$ are adjacent in the 1-subgraph, and that $w_{2}$ and $r$ are adjacent in the 2-subgraph. Besides, by assumption $w_{1}$ has 1-outdegree 2 while $w_{2}$ has 2-outdegree 2. For these reasons, note that the two arcs outgoing from $r$ cannot have the same colour since otherwise $r$ would have 1- or 2-outdegree 2, a contradiction. Then one arc outgoing from $r$ has colour 1 by $\phi$ while the other arc has colour 2, implying that $r$ has both 1- and 2-outdegree 1. But then we necessarily get a contradiction when colouring the arc outgoing from the vertex with outdegree 1 attached to $r$. 
On the contrary, note that if $\phi\left(\overrightarrow{w_{1} \vec{r}}\right)=\phi\left(\overrightarrow{w_{2} \vec{r}}\right)=1$ without loss of generality, then, so that we avoid all contradictions mentioned above, we have to colour 2 all arcs outgoing from $r$. Then $r$ and $r^{\prime}$ are neighbouring vertices in the 2-subgraph, and $r$ has 2-outdegree 2. Since there is a vertex with outdegree 1 attached to $r^{\prime}$, again we cannot colour the two arcs outgoing from $r^{\prime}$ with distinct colours. Then we have to colour 1 the two arcs outgoing from $r^{\prime}$.

Roughly speaking, assuming we are given two arcs $\overrightarrow{a_{1}}$ and $\overrightarrow{a_{2}}$ whose tails necessarily have outdegree 2 in the $\phi_{F}\left(\overrightarrow{a_{1}}\right)$ - and $\phi_{F}\left(\overrightarrow{a_{2}}\right)$-subgraphs, respectively, we can "check" whether $\phi_{F}\left(\overrightarrow{a_{1}}\right)=\phi_{F}\left(\overrightarrow{a_{2}}\right)$. Namely, take a copy of $\overrightarrow{G^{r}}$ and "replace" the arcs $\overrightarrow{w_{1} r}$ and $\overrightarrow{w_{2} r}$ with $\overrightarrow{a_{1}}$ and $\overrightarrow{a_{2}}$, respectively. We refer to this operation as collecting $\overrightarrow{a_{1}}$ and $\overrightarrow{a_{2}}$ (with some copy of $\overrightarrow{G^{r}}$ ). According to Claim 2, the arc-colouring $\phi_{F}$ cannot then be extended to the collecting gadget if $\phi_{F}\left(\overrightarrow{a_{1}}\right) \neq \phi_{F}\left(\overrightarrow{a_{2}}\right)$. Recall further that if $\phi_{F}\left(\overrightarrow{a_{1}}\right)=\phi_{F}\left(\overrightarrow{a_{2}}\right)$, then all of the arcs outgoing from the tail of the output of the collecting gadget have colour $\phi_{F}\left(\overrightarrow{a_{1}}\right)$, and the tail of the output thus has $\phi_{F}\left(\overrightarrow{a_{1}}\right)$-outdegree 2 . In some sense, this property means that the output of a collecting gadget "memorizes" the colour used at its two inputs.

To end up the construction of $\overrightarrow{G_{F}}$, proceed as follows. Consider every variable $x_{i}$ of $F$ with $i \in\{1,2, \ldots, n\}$, and let $\overrightarrow{o_{1}}, \overrightarrow{o_{2}}, \ldots, \overrightarrow{o_{n_{i}}}$ denote the $n_{i}$ arcs of $\overrightarrow{G_{F}}$ representing the membership of $x_{i}$ to some clause, where $n_{i}$ is the number of clauses that contain $x_{i}$. More precisely, these arcs are of the form $\overrightarrow{u_{j} v_{i^{\prime}, j}}$, where $i^{\prime} \in\{1,2,3\}$ and $j \in\{1,2, \ldots, m\}$, and $x_{i}$ is the $i^{\prime}$ th variable of $C_{j}$. Recall further that if any of these arcs $\vec{o}$ is coloured, say, 1 by $\phi_{F}$, then the tail of $\vec{o}$ has 1-outdegree 2. Start by collecting $\overrightarrow{o_{1}}$ and $\overrightarrow{o_{2}}$ with a copy $\overrightarrow{G_{1}}$ of $\overrightarrow{G^{r}}$. Then collect the output of $\overrightarrow{G_{1}}$ and $\overrightarrow{o_{3}}$ with a new copy $\overrightarrow{G_{2}}$ of $\overrightarrow{G^{r}}$. Then collect the output of $\overrightarrow{G_{2}}$ and $\overrightarrow{o_{4}}$ with a new copy $\overrightarrow{G_{3}}$ of $\overrightarrow{G^{\curlyvee}}$. And so on. This procedure uses $n_{i}-1$ copies of $\overrightarrow{G^{\curlyvee}}$.

We claim that we have the desired equivalence between nae-satisfying $F$ and finding a locally irregular 2-arc-colouring $\phi_{F}$ of $\overrightarrow{G_{F}}$. If $\phi_{F}$ exists, then for each clause $C_{j}=\left(x_{i_{1}} \vee x_{i_{2}} \vee x_{i_{3}}\right)$, one arc of $u_{j} v_{1, j}, u_{j} v_{2, j}, u_{j} v_{3, j}$ has some colour by $\phi_{F}$ while the other two arcs have the other colour (Claim 1). Besides, this arc-colouring, because of the collecting gadgets, has the property that all arcs corresponding to the membership of a same variable to some clauses have the same colour (Claim 2). Assuming having $\phi_{F}\left(\overrightarrow{u_{j} v_{i^{\prime}, j}}\right)=1\left(\operatorname{resp} . \phi_{F}\left(\overrightarrow{u_{j} v_{i^{\prime}, j}}\right)=2\right)$ simulates the fact that the $i^{\prime}$ th variable of $C_{j}$ is set to true (resp. false), we can directly deduce a truth assignment nae-satisfying $F$ from $\phi_{F}$, and vice-versa. This completes the proof.

\section{Conclusion and open questions}

Conjecture 1 remains the most important open question at the moment. Maybe the strategy we have proposed in Section 3 could be refined to slightly improve 
Theorem 8, e.g. by showing that every oriented graph can be decomposed into two subgraphs with irregular chromatic index at most 2 and 3, respectively, or two subgraphs with irregular chromatic index at most 2 plus some isolated arcs, etc. However, we do not think that Conjecture 1 can be tackled using this method.

In particular, it is worth mentioning that Theorem 4 is tight since there are acyclic oriented graphs with irregular chromatic index exactly 3 (so we cannot improve the bound in Theorem 8 from 6 to 4 by just refining Theorem 4 ). To be convinced of this statement, one just has to note that LOCALLY IRREGULAR 2-ARC-COLOURING remains NP-complete when restricted to acyclic oriented graphs as it can be easily checked that the reduction in the proof of Theorem 9 actually provides acyclic oriented graphs.

It is worth adding that we were not able to prove that Conjecture 1 holds when restricted to tournaments. Although the question can be handled easily for some restricted families of tournaments, e.g. transitive tournaments (which are locally irregular), we could not find any argument for the general case. Until a proof of Conjecture 1 is exhibited, which would solve the problem, we raise the following weaker conjecture.

Conjecture 13. For every tournament $\vec{T}$, we have $\chi_{\text {irr }}^{\prime}(\vec{T}) \leq 3$.

The authors thank the anonymous referees for their careful reading of a previous version of the current paper. The first author was supported by ERC Advanced Grant GRACOL, project no. 320812.

\section{References}

[1] O. Baudon, J. Bensmail, J. Przybyło, and M. Woźniak. On decomposing regular graphs into locally irregular subgraphs. European Journal of Combinatorics, 49:90-104, 2015.

[2] G. Chartrand, M.S. Jacobson, J. Lehel, O.R. Oellermann, S. Ruiz, and F. Saba. Irregular networks. Congressus Numerantium, 64:197-210, 1988.

[3] R. Diestel. Graph Theory, volume 173 of Graduate Texts in Mathematics. Springer-Verlag, Heidelberg, third edition, 2005.

[4] M.R. Garey and D.S. Johnson. Computers and Intractability: A Guide to the Theory of NP-Completeness. W.H. Freeman, 1979.

[5] W. Imrich and S. Klavžar. Product Graphs: Structure and Recognition. Wiley-Interscience, New York, 2000.

[6] M. Karoński, T. Łuczak, and A. Thomason. Edge weights and vertex colours. Journal of Combinatorial Theory, Series B, 91(1):151-157, 2004. 